\title{
Travel Time Reliability Measurement of Urban Rail Transit System based on Kernel Density Estimation
}

\author{
Weijia Li, Ke Qiao and Peng Zhao \\ School of Traffic and Transportation \\ Beijing Jiaotong University \\ Beijing, China \\ 11120863@bjtu.edu.cn,09114218@bjtu.edu.cn,pzhao@bjtu.edu.cn
}

\begin{abstract}
Travel time reliability is of high importance for passengers and transit agencies in the urban rail transit system. The travel time reliability models of OD pairs, line and network were established, and a short discussion on influence factors of travel time reliability was provided. Based on the real urban rail AFC data, the distribution of travel time was analyzed and a methodology based on kernel density estimation was presented to evaluate travel time reliability. Beijing urban rail transit system was used as a case study. It concluded that the travel time reliability is related to journey distance, interchange times and travel time segment.
\end{abstract}

Keywords-urban rail transit; travel time reliability; kernel density estimation

\section{INTRODUCTION}

Travel time is an important part of passenger travel demand. Passengers not only hope travel time is short, but also hope that travel time is reliable. Travel time reliability has become an important basis for passenger travel mode choice and route choice, and it is also a significant index to evaluate the service quality of rail transit system. For commuters and students, their departure time is relatively fixed. And commuters also have the expected travel time for a certain OD pair, while travel time fluctuation will affect the passengers' travel plans. Compared with the private car and bus, urban rail transit has exclusive right of way, its reliability is high[1]. But with the growing of passenger flow, the contradiction between the rail transit capacity and demand is gradually prominent. Especially in the morning peak, some stations are too crowd and passengers are unable to board the first train, passenger travel time cannot be guaranteed completely. Therefore, according to the actual condition of rail transit network, the accurate calculation of passenger travel time reliability of rail transit system has important significance.

\section{TRAVEL TIME RELIABILITY ANALYSIS}

\section{A. Influence factors of travel time reliability}

Passenger travel time is the entire time they spend from origin to destination. Different passengers' travel time usually have some fluctuations. Fig. 1 is the fluctuation of Xizhimen to Dongzhimen and Pingguoyuan to Dongdan of Beijing rail transit in one day, the line is the passengers average travel time and the asterisk is the passenger actual travel time. It can be seen that passenger travel time has obvious fluctuation in different time segment. Travel time reliability is used to reflect the variability of travel time, it refers to the probability of travel success from the origin to the destination in specific service level. Passengers will make the travel plan according to the travel experience or travel information supported by the operator, and decide when to departure. Therefore, travel time reliability is very important.

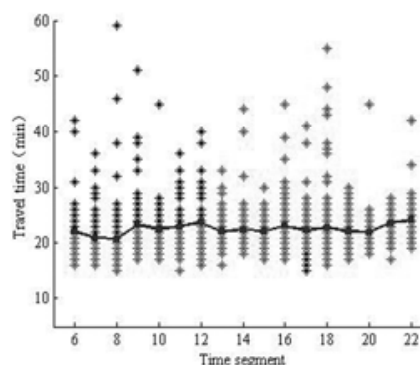

(1) Xizhimen-Dongzhimen

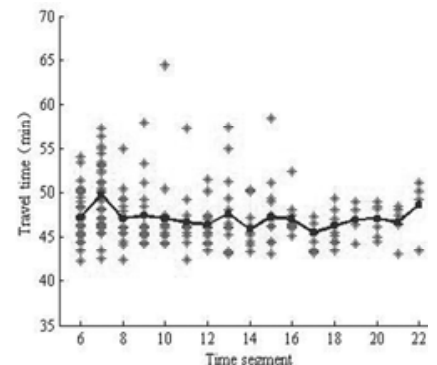

(2) Pingguoyuan-Dongdan
Figure 1. The diagram of travel time fluction

Passenger travel process is expressed in Fig. 2 and Fig. 3. Fig. 3 is with interchange, that the additional interchange walk time and wait time are increased. From the figures, we can see the total travel time can be divided into several components, AWKT means access walk time from entrance to platform, PWT means platform wait time, OTT means on train time, TWKT means interchange walk time, TWT means transfer wait time, EWKT means egress walk time from platform to the exit.

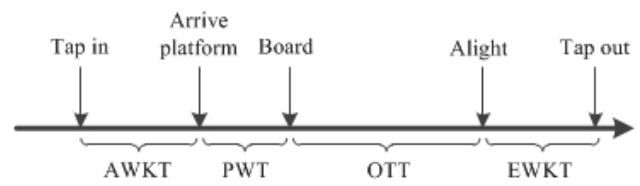

Figure 2. Typical subway travel process without transfer

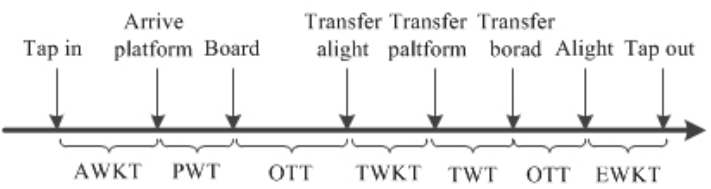

Figure 3. Typical subway travel process with transfer

Travel time reliability is mainly affected by walk time, wait time and on-train time. So in the case study, we will 
analyze the impact of depature time, journey distance and interchange times on travle time reliability in detail.

\section{B. Travel time reliability models}

Based on the existing research [4], the most common measurement indexes of travel time reliability are probability travel time, buffer time index and plan time index. So the travel time reliability models of $O D$ pairs, lines and network were established based on these indexes. Assuming the rail transit network $G$ is composed of $k$ lines, expressed as $G=\left\{l_{1}, l_{2}, \cdots l_{i} \cdots l_{k}\right\}$; One day was divided into three time segments which are morning peak, off-peak and evening peak, expressed as $T=\left\{t_{\text {mor }}, t_{\text {nor }}, t_{\text {eve }}\right\}$; $O D_{m, n}^{G, t_{j}}$ denotes OD pairs between node $m$ and node $n$ at time segment $t_{j}$ in $G$; $O D_{m, n}^{l_{i}, t_{j}}$ denotes OD pairs belongs to line ${ }^{l_{i}}$ at time segment $t_{j}$.

\section{1) Travel time reliability model of $O D$ pairs}

\section{a) Probability travel time}

For fixed OD pairs, assuming that passenger travel time obeys a certain distribution, $f(t)$ is the probability density function of travel time, $\alpha$ is confidence level, travel time reliability is expressed as:

$$
P\left(T_{O D_{m, n}^{G, t_{j}}} \leq T_{O D_{m, n}^{G, t_{j}}}^{\alpha}\right)=1-\alpha
$$

Where, $T_{O D_{m, n}^{G, t_{j}}}^{\alpha}$ is the probability travel time, or the percentile travel time, and shows that the probability that passenger travel time is lower than $T^{\alpha D_{m, n}^{G, t_{j}}}$ at $t_{j}$ is $1-\alpha$. If probability density function is known, we can get different travel time $T^{\prime}$ of different confidence level, $\alpha$ is usually $5 \%$ or $10 \%$, namely probability travel time is $95 \%$ percentile travel time or $90 \%$ percentile travel time, and we use the $95 \%$ percentile travel time. Due to different OD pairs have different journey time, probability travel time cannot be used to compare the differences among different paths.

\section{b) Buffer time coefficient}

In order to avoid travel delay, passengers usually add some buffer time, they will depature earlier to deal with travel time fluctuation. Buffer time is the increased travel time based on the average travel time of OD pairs. Assuming that probability travel time is the maximum travel time passengers can accept, the buffer time coefficient can be expressed as:

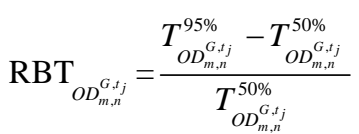

Where, $T^{50 \%} D_{m, n}^{G, t_{j}}$ is passenger average travel time. $\mathrm{RBT}_{O D}$

$T_{O D_{m, n}^{G, t_{j}}}$ can be used to compare the differences among different paths, the bigger the coefficient is , the lower the reliability is.

\section{c) Plan time coefficient}

Plan time is the total travel time including buffer time which passengers expect. Similar with the above, set the probability travel time is the maximum travel time passenger can accept, this coefficient is the ratio of probability travel time and shortest travel time.

$$
\operatorname{RPT}_{O D_{m, n}^{G, t_{j}}}=\frac{T_{O D_{m, n}^{G, t_{j}}}^{95 \%}}{T_{\min }}
$$

Where, $T_{\min }$ is the shortest travel time, which is an ideal passenger travel time without waiting and jam. We can approximately use the minimum travel time of surveys as $T_{\min }$. Plan time coefficient can also be used to compare travel time reliability among different paths.

\section{2) Travel time reliability model of lines and network}

In order to evaluate travel time reliability of lines and network, based on travel time distribution of OD pairs, the travel time is weighted by OD volume. Thus we can get the probability travel time of lines. For line $l_{i}$, the weighted probability travel time $L T_{l_{i}}^{\alpha}$ can be expressed as:

$$
L T_{l_{i}}^{\alpha}=\frac{\sum_{m \in l_{i}} \sum_{n \in l_{i}} T_{O D_{m, n}^{i_{i}, t_{j}}}^{\alpha} \cdot f_{O D_{m, n}^{l_{i}, t_{j}}}}{\sum_{m \in l_{i}} \sum_{n \in l_{i}} f_{O D_{m, n}^{i_{i}, t_{j}}}}
$$

Then we can get buffer time coefficient of lines $R B T_{l_{i}}$ which can be used to compare travel time reliability of different lines and measure service level of lines.

$$
R B T_{l_{i}}=\frac{L T_{l_{i}}^{95 \%}-L T_{l_{i}}^{50 \%}}{L T_{l_{i}}^{50 \%}}
$$

Similarly, for the entire network, considering all OD pairs, weighted probability travel time of network can be obtained.

$$
N T_{G}^{\alpha}=\frac{\sum_{m \in G} \sum_{n \in G} T_{O D_{m, n}^{G, t_{j}}}^{\alpha} \cdot f_{O D_{m, n}^{G, t_{j}}}}{\sum_{m \in G} \sum_{n \in G} f_{O D_{m, n}^{G, t_{j}}}}
$$

Then the buffer time coefficient of network is expressed as: 


$$
R B T_{G}=\frac{N T_{G}^{95 \%}-N T_{G}^{50 \%}}{L T_{G}^{50 \%}}
$$

\section{TRAVEL Time Reliability CALCUlate MEthoD}

\section{A. Passenger travel time distribution analysis}

Some OD pairs of Beijing rail transit were selected to do the test. The results show that the values of KolmogorovSmirnov $\mathrm{Z}$ are all zero, and the assumption that the travel time is normal distribution is rejected. Normal probability unit distribution diagrams of some OD pairs were shown in Fig. 4. According to the drawing principle, if the normal probability distribution diagram is crooked in the right corner, it means that the distribution has a tail thicker than the normal distribution and it is skewed distribution, illustrated the passenger travel time is with certain clusters. Most passengers travel time is concentrated in a certain period of time, a small portion of passengers travel time is significantly longer. This also shows that passenger travel time is not completely random, although travel paths and travel information is the same. But passengers have a certain lag for travel information.

Because travel time distribution is not the normal distribution, in order to describe the travel time distribution law more accurately, the Gauss kernel density estimation is used to fit the travel time distribution.

Define abbreviations and acronyms the first time they are used in the text, even after they have been defined in the abstract. Abbreviations such as IEEE, SI, MKS, CGS, sc, dc, and rms do not have to be defined. Do not use abbreviations in the title or heads unless they are unavoidable.

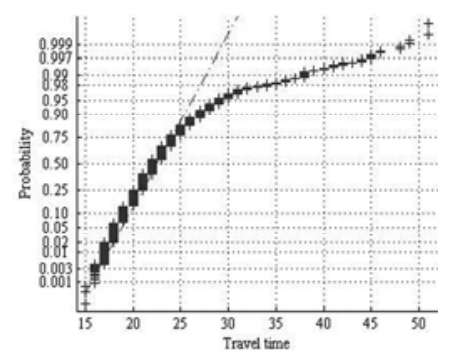

(1) Xizhimen-Dongzhimen

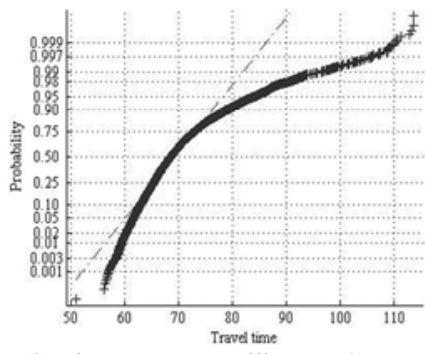

(3) Tiantongyuan-Beijingnanzhan

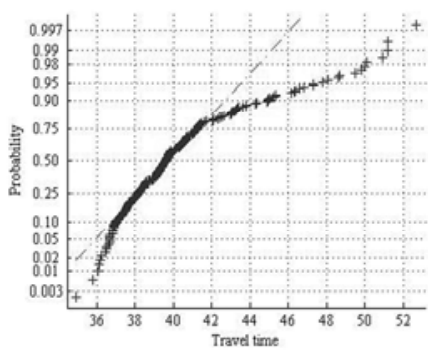

(2) Tiantongyuan-Dongdan

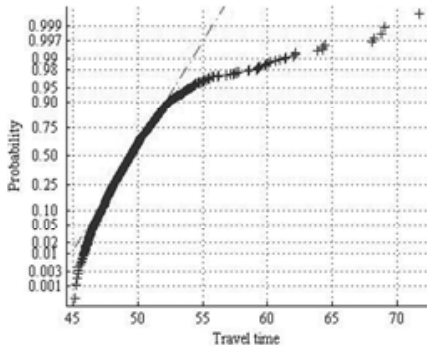

(4) Bagou-Jinsong
Figure 4. OD pairs travel time normal probability Q-Q map

\section{B. Measurement algorithm of travel time reliability based on} kernel density estimation

Kernel density estimation belongs to the category of nonparametric estimation[11], it can estimate the density function even if there is no overall distribution of objects. The definition of Gauss kernel estimation is as follows:

Set $K(\bullet)$ is a given probability density function in $R$. $h_{n}>0$ is a constant related to $n$, when $n \rightarrow \infty, h_{n} \rightarrow \infty$, then the

$$
\hat{f_{n}}(x)=\frac{1}{n h_{n}} \sum_{i=1}^{n} K\left(\frac{x_{i}-x}{h_{n}}\right)
$$

is kernel density estimation of the estimation function $f(x)$, where, $h_{n}$ is bandwidth, $n$ is the sample size, $x_{1}, x_{2} \cdots x_{n}$ is a set of independent discrete random sample. Kernel density estimation mainly depends on the kernel function $K(\bullet)$ and the bandwidth $h_{n}$. It is generally believed that the choice of kernel function is not sensitive in kernel density estimation, the Gauss kernel function is selected in our research, the Gauss kernel function is expressed as:

$$
K(u)=\frac{1}{\sqrt{2 \pi}} e^{-\frac{u^{2}}{2}}, u \in(-\infty,+\infty)
$$

The choice of bandwidth has a large impact on the fitting effect of kernel density estimation. How to choose the appropriate bandwidth is key to a successful application of kernel density estimation. Assuming that each sample approximately obey the normal distribution, the optimal bandwidth can be calculated using the following formula:

$$
h_{n}=1.06 \sigma n^{-0.2}
$$

Therefore, the process of travel time reliability measurement is as follows:

Step1: get cumulative distribution function based on density function estimation, $F(t)=\int_{-\infty}^{t} \hat{f(t) d t}$;

Step2: calculate the inverse function of cumulative distribution function $F^{-1}(t)$;

Step3: according to the inverse function, calculate $T_{O D_{m, n}^{G, t_{j}}}^{\alpha}$ and get $\mathrm{RBT}_{O D_{m, n}^{G, t_{j}} \text { and }} \mathrm{RPT}_{O D_{m, n}^{G, t_{j}}}$.

\section{EXPERIMENT ANALYSIS}

\section{A. Data Description}

The Auto Fare Collection (AFC) system is used in the rail transit at present, the AFC can obtain the time passenger entering and exiting the station. Ten OD pairs of Beijing rail transit were selected according to journey distance, interchange times, and multiple paths, as shown in table 1 , and we get data of one day of these OD pairs. Before analyzing the data, the obvious wrong data was eliminated. 
TABLE I. SELECTED OD PAIRS

\begin{tabular}{|c|c|c|c|}
\hline & $\begin{array}{c}\text { Without } \\
\text { interchange }\end{array}$ & With interchange & Multi paths \\
\hline $\begin{array}{l}\text { Short } \\
\text { distance }\end{array}$ & $\begin{array}{l}\text { Xizhimen- } \\
\text { Dongzhimen and } \\
\text { Xizhimen-Xidan } \\
\text { Tiantongyuan- }\end{array}$ & & $\begin{array}{l}\text { Dongzhongsi- } \\
\text { Xuanwumen }\end{array}$ \\
\hline $\begin{array}{l}\text { Medium } \\
\text { distance }\end{array}$ & $\begin{array}{l}\text { Dongdan and } \\
\text { Pingguoyuan- } \\
\text { Dongdan }\end{array}$ & $\begin{array}{l}\text { Chuanmeidaxue- } \\
\text { Junshibowuguan }\end{array}$ & $\begin{array}{l}\text { Jianguomen- } \\
\text { Lishuiqiao }\end{array}$ \\
\hline $\begin{array}{l}\text { Long } \\
\text { distance }\end{array}$ & Bagou-Jinsong & $\begin{array}{l}\text { Tiantongyuan- } \\
\text { Junshibowuguan }\end{array}$ & $\begin{array}{l}\text { Tiantongyuan- } \\
\text { Beijingnanzhan }\end{array}$ \\
\hline
\end{tabular}

\section{B. Calculation values of travel time reliability}

In order to calculate travel time reliability indexes of each OD pair in all day, the travel time probability density formula is calculated first based on the Gauss kernel density .Having the Xizhimen-Dongzhimen OD for example, the distribution curve based on Gauss kernel density estimation is as Fig. 5, it shows that using this method can fit the travel time probability distribution better.

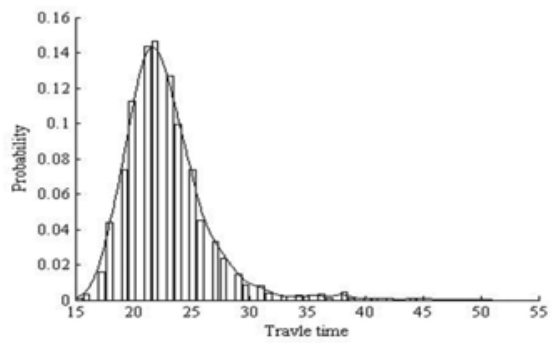

Figure 5. Travel time probability distribution diagram of XizhimenDongzhimen

The calculation results are shown in Table 2, we can compare travel time reliability between different OD pairs.

TABLE II. INDEXES OF OD PAIRS TRAVEL TIME RELIABILITY IN THE

\begin{tabular}{lccccc} 
& \multicolumn{5}{c}{ WHOLE DAY } \\
\hline & $\begin{array}{l}T_{O D_{m, n}^{G, t_{j}}}^{50 \%} \\
(\mathbf{m i n})\end{array}$ & $\begin{array}{c}T_{O D_{m, n}^{G, t_{j}}}^{95 \%} \\
(\mathbf{m i n})\end{array}$ & $\begin{array}{c}T_{\text {min }} \\
(\mathbf{m i n})\end{array}$ & $\mathrm{RBT}_{O D_{m, n}^{G, t_{j}}}$ & $\mathrm{RPT}_{O D_{m, n}^{G, t_{j}}}$ \\
\hline $\begin{array}{l}\text { Xizhimen- } \\
\text { Dongzhimen }\end{array}$ & 22.00 & 29.09 & 15.00 & 0.32 & 1.94 \\
$\begin{array}{l}\text { Xizhimen-Xidan } \\
\text { Tiantongyuan- }\end{array}$ & 17.03 & 24.20 & 13.03 & 0.42 & 1.86 \\
$\begin{array}{l}\text { Dongdan } \\
\begin{array}{l}\text { Pingguoyuan- } \\
\text { Dongdan }\end{array}\end{array}$ & 39.62 & 47.63 & 34.90 & 0.20 & 1.36 \\
$\begin{array}{l}\text { Bagou-Jinsong } \\
\text { Chuanmeidaxue- }\end{array}$ & 49.45 & 54.04 & 42.43 & 0.16 & 1.27 \\
$\begin{array}{l}\text { Junshibowuguan } \\
\text { Tiantongyuan- }\end{array}$ & 49.25 & 58.15 & 45.15 & 0.10 & 1.20 \\
$\begin{array}{l}\text { Junshibowuguan } \\
\begin{array}{l}\text { Dazhongsi- } \\
\text { Xuanwumen }\end{array}\end{array}$ & 63.74 & 73.75 & 57.35 & 0.16 & 1.29 \\
$\begin{array}{l}\text { Jianguomen- } \\
\text { Lishuiqiao }\end{array}$ & 43.35 & 43.90 & 24.32 & 0.32 & 1.80 \\
$\begin{array}{l}\text { Tiantongyuan- } \\
\text { Beijingnanzhan }\end{array}$ & 68.60 & 84.06 & 50.95 & 0.23 & 1.65 \\
\hline
\end{tabular}

\section{CONCLUSION}

Due to the growing demand and the increasingly serious congestion in the peak times in the urban rail transit system, travel time reliability has attracted the attention of passengers and transit agencies. In this paper, travel time reliability models of OD pairs, lines and network were developed. The travel time distribution is the basis of the model and the distribution is not normal distributed through KolmogorovSmirnov test, it has a significant tail characteristics. So an algorithm based on kernel density estimation was proposed to evaluate the travel time reliability. Through the case analysis, it found that travel time reliability can be affected by the depature time, journey distance and interchange times. Because we don't have the enough data of the entire network, how to evaluate travel time reliability of lines and network remains to be further in-depth excavation.

\section{ACKNOWLEDGMENT}

This work was sponsored by Research Fund for the Doctoral Program of Higher Education of China (NO. 20120009110016).

\section{REFERENCES}

[1] Vuchic VR. Urban Transit: Operations Planning and Economics. Beijing China Railway Publishing House. 2012.

[2] Chang JS. Assessing Travel Time Reliability in Transport Appraisal. Journal of Transport Geography. 2010; 18(3): 419-425.

[3] Chen Kun, Yu Lei. Route Travel Time Reliability Model Based on Lognormal Sum Distribution. Journal of Beijing Jiaotong University. 2009; 33(6): 36-38.

[4] Li Xian, Wen Huimin, Gao Yong, Chen Kun. Beijing Road Network Rate Travel Time Reliability Evalutaion. Journal of Transportation Systems Engineering and Information Technology. 2007; 7(2): 72-76.

[5] Chen Chenghui. Research on Travel Time Reliability of Multi-mode Public Transport. MS Thesis. Nanjing: Nanjing University of Science and Technology; 2011.

[6] Borjesson M, Eliasson J. On The Use of “Average Delay” As a Measure of Train Reliability. Transportation Research Part A. 2011; 45(3): 171184.

[7] Wang Zhigang, Shi Rong, Gao Weijun. Analysis of Passenger's Walking Time Function at Shanghai Urban Rail Station. Urban Mass Transit. 2010; 13(12): 57-60.

[8] Hao Yong. Study on the Impact of Pedestrian Facilities to Passenger Walking Time at Subway Stations. Railway Transport and Economy. 2009; 31(2): 70-72.

[9] Du Peng, Liu Chao, Liu Zhili. Walking Time Modeling on Transfer Pedestrians in Subway Passages. Journal of Transportation Systems Engineering and Information Technology. 2009; 9(4): 103-108.

[10] Lai Shukun. Research on Income Distribution Model of Urban Rail Transit. MS Thesis. Beijing: Beijing Jiaotong University; 2008.

[11] Ma Shoufeng, He Zhengbing, Zhang Siwei. Approach Based on Risk for Traffic Network Reliability. Systems Engineering -Theory \& Practice. 2010; 30(3): 550-555. 